\title{
Factorization Theorem Connecting the Light-Cone Distribution Amplitudes of Heavy-Flavor Mesons in QCD and Heavy-Quark Effective Theory
}

\author{
Saadi Ishaq, ${ }^{2,3,5, *}$ Yu Jia, ${ }^{2,3, \dagger}$ Xiaonu Xiong, ${ }^{1,4, \$}$ and De-Shan Yang ${ }^{3,2, \S}$ \\ ${ }^{1}$ School of Physics and Electronics, Central South University, Changsha 418003, China \\ ${ }^{2}$ Institute of High Energy Physics, Chinese Academy of Sciences, Beijing 100049, China \\ ${ }^{3}$ School of Physical Sciences, University of Chinese Academy of Sciences, Beijing 100049, China \\ ${ }^{4}$ Institute for Advanced Simulation, Institut für Kernphysik and Jülich Center for Hadron Physics, \\ Forschungszentrum Jülich, D-52425 Jülich, Germany \\ ${ }^{5}$ Department of Physics, School of Natural Sciences (SNS), National University of Sciences and Technology (NUST), \\ Sector H-12, Islamabad 44000, Pakistan
}

(Received 26 June 2020; accepted 24 July 2020; published 21 September 2020)

\begin{abstract}
The light-cone distribution amplitude (LCDA) of a heavy-light meson defined in heavy quark effective theory (HQET) is a fundamental nonperturbative input to account for innumerable $B$ meson exclusive decay and production processes. On the other hand, the conventional heavy-flavored meson LCDA defined in QCD also ubiquitously enters the factorization formula for hard exclusive $B$ production processes. Inspired by the observation that these two LCDAs exhibit the identical infrared behaviors, yet differ in the ultraviolet scale of order $m_{b}$ or greater, we propose a novel factorization theorem for the heavy-light mesons, that the LCDA defined in QCD can be further expressed as a convolution between the LCDA in HQET and a perturbatively calculable coefficient function thanks to asymptotic freedom. This refactorization program can be invoked to fully disentangle the effects from three disparate scales $Q, m_{b}$, and $\Lambda_{\mathrm{QCD}}$ for a hard exclusive $B$ production process, particularly to facilitate the resummation of logarithms of type $\ln Q / m_{b}$ and $\ln m_{b} / \Lambda_{\mathrm{QCD}}$ in a systematic fashion.
\end{abstract}

DOI: 10.1103/PhysRevLett.125.132001

The hard exclusive hadron production is one of the major battlefields of perturbative QCD [1]. If one denotes the hard momentum transfer scale by $Q$, the famous collinear factorization theorem $[2,3]$ demands that the reaction amplitude involving a single hadron can be expressed as the following convolution integral:

$$
\mathcal{M}=\int_{0}^{1} d x T\left(x ; \mu_{Q}\right) \Phi^{\mathrm{QCD}}\left(x ; \mu_{Q}\right)+\mathcal{O}\left(\frac{1}{Q}\right),
$$

up to higher-twist corrections. Here $0 \leq x \leq 1$ signifies the light-cone momentum fraction of the quark inside the hadron. $T$ represents the perturbatively calculable hardscattering kernel, $\Phi^{\mathrm{QCD}}$ denotes the nonperturbative, yet universal, leading-twist light-cone distribution amplitude (LCDA) of the hadron defined in QCD. The factorization scale $\mu_{Q}$ lies between $Q$ and $M \sim \Lambda_{\mathrm{QCD}}$ ( $M$ denotes the hadron mass), which enters both $T$ and $\Phi^{\mathrm{QCD}}$ in a prescribed manner such that the physical amplitude becomes independent of this artificial scale. Specifically,

Published by the American Physical Society under the terms of the Creative Commons Attribution 4.0 International license. Further distribution of this work must maintain attribution to the author(s) and the published article's title, journal citation, and DOI. Funded by SCOAP ${ }^{3}$. the $\mu$ dependence of the QCD LCDA is governed by a celebrated renormalization group equation, usually referred to as the Efremov-Radyushkin-Brodsky-Lepage (ERBL) equation $[4,5]$ :

$\mu_{Q} \frac{d}{d \mu_{Q}} \Phi^{\mathrm{QCD}}\left(x ; \mu_{Q}\right)=\frac{\alpha_{s} C_{F}}{\pi} \int_{0}^{1} d y V_{0}(x, y) \Phi^{\mathrm{QCD}}\left(y ; \mu_{Q}\right)$,

with the color factor $C_{F}=\left(N_{c}^{2}-1\right) /\left(2 N_{c}\right)$, and $N_{c}=3$ being the number of color. The evolution kernel for a helicity-zero meson reads

$V_{0}(x, y)=\left[\frac{x}{y}\left(1+\frac{1}{y-x}\right) \theta(y-x)+\left(\begin{array}{l}x \rightarrow \bar{x} \\ y \rightarrow \bar{y}\end{array}\right)\right]_{+}$,

with $\bar{x} \equiv 1-x$. This equation can facilitate to resum large collinear logarithm of type $\alpha_{s} \ln \left(Q / \Lambda_{\mathrm{QCD}}\right)$ in a typical hard exclusive reaction. Note that the formalism in Eq. (1) applies to any species of hadrons, irrespective of being light or heavy, provided that $Q \gg M \geq \Lambda_{\mathrm{QCD}}$.

Alternative factorization framework also exists if the reaction involves a heavy-flavor meson, such as $B$ exclusive decay process, exemplified by $B \rightarrow \gamma \ell \nu$ [6-8]. By exploiting the hierarchy $m_{b} \gg \Lambda_{\mathrm{QCD}}$, the HQET factorization theorem $[9,10]$ demands that the $B$ exclusive decay amplitude may also be cast into a convolution form: 


$$
\mathcal{M}=\int_{0}^{\infty} d \omega \mathcal{T}\left(\omega, Q, m_{b} ; \mu_{H}\right) \Phi_{+}^{\mathrm{HQET}}\left(\omega ; \mu_{H}\right)+\mathcal{O}\left(\frac{1}{m_{b}}\right),
$$

in the $m_{b} \rightarrow \infty$ limit. Here $\omega$ signifies the light-cone momentum of the light spectator quark inside the $B$ meson. $\mathcal{T}$ again represents the perturbatively calculable hardscattering kernel thanks to asymptotic freedom, $\Phi_{+}^{\mathrm{HQET}}$ denotes the nonperturbative, yet universal, leading-power LCDA of the $B$ meson, with the $b$-quark field defined in the heavy-quark effective theory (HQET) $[11,12]$. For a discussion about the model-independent properties of the $B$ meson LCDA in HQET, see [13]. A peculiar feature of $\Phi_{+}^{\mathrm{HQET}}(\omega)$ is that its positive Mellin moments become UV divergent, where one usually imposes a UV cutoff $\Lambda$ to regularize. The $m_{b}$ dependence is entirely encoded in $\mathcal{T}$ but not in $\Phi_{+}^{\mathrm{HQET}}$. Unlike collinear factorization, here one does not distinguish the scales $Q$ and $m_{b}$ in the hard-scattering kernel. The dependence of $\mathcal{T}$ and $\Phi_{+}^{\mathrm{HQET}}$ on the factorization scale $\mu_{H}$, which lies between $m_{b}$ and $\Lambda_{\mathrm{QCD}}$, conspires to counterbalance each other in the physical amplitude.

The scale dependence of the $B$ meson LCDA defined in the HQET side is controlled by the famous Lange-Neubert evolution equation [14]:

$$
\begin{aligned}
\mu_{H} & \frac{d}{d \mu_{H}} \phi_{+}^{\mathrm{HQET}}\left(\omega ; \mu_{H}\right) \\
& =-\frac{\alpha_{s} C_{F}}{4 \pi} \int_{0}^{\infty} d \omega^{\prime} \gamma_{+}\left(\omega, \omega^{\prime} ; \mu_{H}\right) \phi_{+}^{\mathrm{HQET}}\left(\omega^{\prime} ; \mu_{H}\right),
\end{aligned}
$$

where the one-loop anomalous dimension $\gamma_{+}$reads

$$
\begin{aligned}
\gamma_{+}\left(\omega, \omega^{\prime} ; \mu_{H}\right)= & \left(\Gamma_{\text {cusp }}^{(1)} \ln \frac{\mu_{H}}{\omega}-2\right) \delta\left(\omega-\omega^{\prime}\right) \\
& -\Gamma_{\text {cusp }}^{(1)} \omega\left[\frac{\theta\left(\omega^{\prime}-\omega\right)}{\omega^{\prime}\left(\omega^{\prime}-\omega\right)}+\frac{\theta\left(\omega-\omega^{\prime}\right)}{\omega\left(\omega-\omega^{\prime}\right)}\right]_{+},
\end{aligned}
$$

with $\Gamma_{\text {cusp }}^{(1)}=4$. The explicit occurrence of $\ln \mu_{H}$ in the anomalous dimension might look peculiar. This evolution equation can be employed to resum large soft logarithms of form $\alpha_{s} \ln ^{n}\left(m_{b} / \Lambda_{\mathrm{QCD}}\right)(n=1,2)$.

Apart from numerous $B$ exclusive decay modes, we emphasize that the HQET factorization formalism (4) can also be fruitfully applied to exclusive $B$ production processes. Various exclusive $D^{ \pm}$production processes have been investigated long ago at tree level, essentially following the ansatz of (4), yet coined with a different terminology: heavy-quark recombination mechanism [15]. It is worth noting that HQET factorization framework presents a successful and economic account for the $D^{+} / D^{-}$production asymmetry observed in various Fermilab fixed target experiments $[16,17]$. Very recently, the exclusive processes $W \rightarrow B\left(D_{s}\right)+\gamma$ have been calculated to order $\alpha_{s}$ in the context of HQET factorization [18]. We note that some exclusive channels of $W, Z$ radiative decays into heavyflavor mesons have previously been investigated in the standard QCD collinear factorization [19].

One interesting question may be naturally posed: for a hard exclusive $B$ production process with scale hierarchy $Q \gg m_{b} \gg \Lambda_{\mathrm{QCD}}$, since both light-cone factorization (1) and HQET factorization (4) appear to be applicable, how can one manage to make the most optimized predictions?

Surely both factorization approaches are based upon solid theoretical ground, nevertheless each of which has its own strength and weakness. As mentioned before, a notable merit of the light-cone approach is that large collinear logarithm $\ln Q / m_{b}$ can be efficiently resummed by considering $m_{b}$ as an IR scale. However, an apparent shortcoming of this approach is that the characteristic feature of the heavy-flavor meson is not adequately utilized, and the phenomenological constraints on $B$ meson LCDA defined in QCD are also limited. From theoretical angle, it is evident that the QCD LCDA cannot be entirely nonperturbative, since it entails the hard scale $m_{b}$, and it is definitely desirable if this perturbative effect can be explicitly separated from the $B$ meson QCD LCDA. On the other hand, the strength of the HQET factorization is that the heavy-quark nature of the $B$ meson has been fully exploited by treating $m_{b}$ as a UV scale. Moreover, much phenomenological knowledge on the $B$ meson LCDA defined in HQET has been gleaned based on intensive investigations on numerous $B$ decay processes over the past two decades. The weakness of this approach for exclusive $B$ production is that the hard-scattering kernel involves two disparate scales $Q$ and $m_{b}$, and the large collinear logarithm may potentially ruin the convergence of perturbative expansion.

The goal of this Letter is to show that these two factorization approaches can be fruitfully combined to make optimized predictions for hard exclusive heavy meson production. The key is to establish a factorization formula connecting two types of $B$ meson LCDAs defined in both QCD and HQET. As we shall see, through the refactorization program, the effects from three disparate scales, $Q, m_{b}$, and $\Lambda_{\mathrm{QCD}}$, can be fully disentangled.

We first recapitulate how the $B$ meson LCDAs are framed in QCD and HQET. For simplicity, we will concentrate on the $\bar{B}$ meson composed of a $b$ quark and a light spectator antiquark $\bar{q}$. Let a timelike four-vector $v^{\mu}$ represent the four-velocity of the $\bar{B}$ meson, which satisfies $P^{\mu}=m_{B} v^{\mu}$ and $v^{2}=1$. For convenience we also introduce a reference null vector $n^{\mu}$ satisfying $n^{2}=0$. The explicit definitions for both LCDAs then become

$$
\begin{aligned}
& \Phi^{\mathrm{QCD}}\left(x, \mu_{Q}\right) \equiv f_{B} \phi^{\mathrm{QCD}}\left(x, \mu_{Q}\right) \\
& \quad=-i \int \frac{d z^{-}}{2 \pi} e^{i x P^{+} z^{-}}\left\langle 0\left|\bar{q}(z)[z, 0] \not h \gamma_{5} b(0)\right| \bar{B}(P)\right\rangle,
\end{aligned}
$$




$$
\begin{aligned}
& \Phi_{+}^{\mathrm{HQET}}\left(\omega, \mu_{H}\right) \equiv \hat{f}_{B} \phi_{+}^{\mathrm{HQET}}\left(\omega, \mu_{H}\right) \\
& \quad=\frac{-i}{m_{B} v^{+}} \int \frac{d t}{2 \pi} e^{i \omega t}\left\langle 0\left|\bar{q}(z)[z, 0] \not h \gamma_{5} h_{v}(0)\right| \bar{B}(v)\right\rangle,
\end{aligned}
$$

where $z^{\mu}=z^{-} n^{\mu}$ is also a null coordinate vector, $t=v \cdot z$. We have also defined $P^{+}=n \cdot P$ and $v^{+}=n \cdot v \cdot[z, 0]$ represents the lightlike gauge link, inserted to ensure gauge invariance. $x$ in Eq. (7) designates the light-cone momentum fraction of the light spectator quark $\bar{q}$. (In Ref. [20], the author adopted the same $\omega$ in the definition of the Fourier transformed QCD LCDA as in the definition of HQET LCDA; here we choose the light-cone fraction $x$ as the Fourier conjugate variable to respect the tradition.) The QCD decay constant $f_{B}$ can be factored onto the HQET decay constant $\hat{f}_{B}$ by integrating out hard quantum fluctuation of order $m_{b}$ through the perturbative matching [21,22]:

$f_{B}=\hat{f}_{B}\left(\mu_{H}\right)\left[1-\frac{\alpha_{s} C_{F}}{4 \pi}\left(3 \ln \frac{\mu_{H}}{m_{b}}+2\right)\right]+\mathcal{O}\left(\alpha_{s}^{2}\right)$.

Notice the only difference of two LCDAs in Eq. (7) is that the $b$ quark field is defined in QCD for the former, while defined in HQET for the latter. Obviously these two LCDAs have drastically different ultraviolet behavior, as is evident in the completely different evolution of Eqs. (2) and (5). Nevertheless, it is crucial to observe that these two objects possess exactly the identical infrared behavior, since HQET faithfully reproduces the IR aspects of QCD. Since the perturbative scale $m_{b}$ is still encompassed in $\Phi^{\mathrm{QCD}}$, it sounds appealing to explicitly factor this shortdistance effect out of the QCD LCDA. Conceivably, this scale separation can be achieved through the following refactorization program:

$\Phi^{\mathrm{QCD}}\left(x, \mu_{Q}\right)=\int_{0}^{\infty} d \omega Z\left(x, \omega, m_{b} ; \mu_{Q}, \mu_{H}\right) \Phi_{+}^{\mathrm{HQET}}\left(\omega, \mu_{H}\right)$.

The coefficient function $Z$ captures all the effect of order $m_{b} \gg \Lambda_{\mathrm{QCD}}$ and thus can be computed in perturbation theory thanks to asymptotic freedom. It can be organized as

$$
\begin{aligned}
Z\left(x, \omega, m_{b} ; \mu_{Q}, \mu_{H}\right)= & Z^{(0)}\left(x, \omega, m_{b}\right) \\
& +\frac{\alpha_{s} C_{F}}{4 \pi} Z^{(1)}\left(x, \omega, m_{b} ; \mu_{Q}, \mu_{H}\right) \\
& +\mathcal{O}\left(\alpha_{s}^{2}\right) .
\end{aligned}
$$

The physical picture underlying Eq. (9) may also be lucidly envisioned in the context of strategy of region [23]. When computing the perturbative correction to the QCD LCDA in Eq. (7a), the loop momentum flowing into the $b$ can be partitioned into either hard $\left(l^{\mu} \sim m_{b}\right)$ or soft $\left(l^{\mu} \ll m_{b}\right)$ regions. It is the soft region that is exactly responsible for the contribution to the HQET LCDA defined in Eq. (7b), which is also equivalent to taking $m_{b} \rightarrow \infty$ limit prior to conducting the loop integration. Therefore, the $Z$ function in Eq. (9), which just accounts for the difference between these two LCDAs, receives contribution solely from the hard loop region; therefore, it can be accessed in perturbation theory thanks to the asymptotic freedom.

Before proceeding, we pause to remark that the refactorization program here is in spirit analogous to the factorization of the LCDA of the doubly flavored heavy quarkonium, $B_{c}$, into the nonperturbative local NRQCD matrix element multiplied with the perturbatively calculable coefficient function [24,25]. It is amusing to point out that the structure of Eq. (9) also looks similar to the factorization formula that links the quasi and light-cone parton distributions, which has been recently formulated in the context of the large-momentum effective theory by Ji [26]. There the light-cone distribution function has the support $(0,1)$, while the quasidistribution has unbounded support $(-\infty,+\infty)$. Of course, a notable difference between these two situations is that both $B$ meson distribution amplitudes in our case are light-cone correlators.

Determination of the $Z$ function can be best fulfilled via the standard perturbative matching procedure. Since the $Z$ factor is insensitive to the IR physics, one can freely replace the nonperturbative $\bar{B}$ meson by a fictitious one, i.e., a free $b \bar{q}$ pair, and compute the corresponding $\Phi^{\mathrm{QCD}}$ and $\Phi_{+}^{\mathrm{HQET}}$ in perturbation theory:

$$
\begin{aligned}
\Phi^{\mathrm{QCD}}\left(x, \mu_{Q}\right)= & \Phi^{\mathrm{QCD}(0)}\left(x, \mu_{Q}\right) \\
& +\frac{\alpha_{s} C_{F}}{4 \pi} \Phi^{\mathrm{QCD}(1)}\left(x, \mu_{Q}\right)+\mathcal{O}\left(\alpha_{s}^{2}\right), \\
\Phi_{+}^{\mathrm{HQET}}\left(\omega, \mu_{H}\right)= & \Phi_{+}^{\mathrm{HQET}(0)}\left(\omega, \mu_{H}\right) \\
& +\frac{\alpha_{s} C_{F}}{4 \pi} \Phi_{+}^{\mathrm{HQET}(1)}\left(\omega, \mu_{H}\right)+\mathcal{O}\left(\alpha_{s}^{2}\right) .
\end{aligned}
$$

One is then able to solve Eq. (9) to deduce the $Z$ factor iteratively, order by order in $\alpha_{s}$. Note this matching procedure is similar to deducing the perturbative $Z$ factor that connects the quasi parton distribution functions and light-cone parton distributions [27].

One can set up the kinematic configuration for $|b \bar{q}\rangle$ at his disposal. For example, one may simply follow [18] to choose a static $b$ and a moving $\bar{q}$. Fortunately, by modeling the $B$ meson as a free $b \bar{q}$ pair with vanishing relative motion, Bell and Feldmann had already computed $\Phi^{\mathrm{QCD}}$ and $\Phi_{+}^{\mathrm{HQET}}$ through order $\alpha_{s}$ a decade ago [28]. Consequently, based on their results, we can directly extract the intended order- $\alpha_{s}$ part of the $Z$ function. There the spectator light quark $\bar{q}$ is endowed with a nonvanishing constitute mass $m_{q}$, which serves to regularize the mass (collinear) singularity. At lowest order, both perturbative 
LCDAs of the fictitious $\bar{B}$ meson are simply $\delta$ functions [28]:

$$
\begin{gathered}
\phi^{\mathrm{QCD}(0)}(x)=\delta\left(x-x_{0}\right), \\
\phi_{+}^{\mathrm{HQET}(0)}(\omega)=\delta\left(\omega-m_{q}\right),
\end{gathered}
$$

with $x_{0}=m_{q} / m_{B}$ and $m_{B}=m_{b}+m_{q}$. From Eq. (9), one readily finds that the tree-level $Z$ factor is also a simple $\delta$ function.

$$
Z^{(0)}\left(x, \omega, m_{b}\right)=\delta\left(x-\frac{\omega}{m_{b}+\omega}\right) .
$$

Reassuringly, the perturbative $Z$ factor is absent of the IR scale $m_{q}$. The $\delta$ function guarantees that the $\omega$ with support $(0, \infty)$ is monotonically mapped onto $x$ with support $(0,1)$.

By solving Eq. (9) to next-to-leading order, we then obtain the $Z$ factor of order $\alpha_{s}$ (the substitution of $m_{q}$ to $\omega$ is due to the striping off the LCDAs $\phi^{\mathrm{QCD}(0)}(x)$ and $\phi_{+}^{\mathrm{HQET}(0)}(\omega)$ from the convolution in order to reveal the $Z$ factor at the next-to-leading order of $\alpha_{s}$ ):

$$
\begin{aligned}
Z^{(1)}\left(x, \omega, m_{b} ; \mu_{Q}, \mu_{H}\right)= & \left.\phi^{\mathrm{QCD}(1)}\left(x, \mu_{Q}\right)\right|_{m_{q} \rightarrow \omega} \\
& -\left.\frac{m_{b}}{(1-x)^{2}} \phi_{+}^{\mathrm{HQET}(1)}\left(\frac{m_{b} x}{1-x}, \mu_{H}\right)\right|_{m_{q} \rightarrow \omega} \\
& -\left(3 \ln \frac{\mu_{H}}{m_{b}}+2\right) Z^{(0)}\left(x, \omega, m_{b}\right) .
\end{aligned}
$$

By construction, the $Z$ factor automatically obeys the ERBL equation and LN equation.

Plugging the explicit order- $\alpha_{s}$ expressions for two LCDAs [28] into Eq. (14), we end up with

$$
\begin{aligned}
Z^{(1)}\left(x, \omega, m_{b} ; \mu_{Q}, \mu_{H}\right)= & 2\left\{\left(\ln \frac{\mu_{Q}^{2}}{\left(m_{b}+\omega\right)^{2}\left(x_{\omega}-x\right)^{2}}-1\right)\left[\left(1+\frac{1}{x_{\omega}-x}\right) \frac{x}{x_{\omega}} \theta\left(x_{\omega}-x\right)+\left(\begin{array}{c}
x \leftrightarrow \bar{x} \\
x_{\omega} \leftrightarrow \bar{x}_{\omega}
\end{array}\right)\right]\right\}_{[x]+} \\
& +4\left\{\frac{x(1-x)}{\left(x-x_{\omega}\right)^{2}}\right\}_{[x]++}+2 \delta^{\prime}\left(x-x_{\omega}\right)\left(2 x_{\omega}\left(1-x_{\omega}\right) \ln \frac{x_{\omega}}{1-x_{\omega}}+2 x_{\omega}-1\right) \\
& -\omega_{x} \frac{d \omega_{x}}{d x}\left\{2\left[\left(\ln \left[\frac{\mu_{H}^{2}}{\left(\omega_{x}-\omega\right)^{2}}\right]-1\right)\left(\frac{\theta\left(\omega-\omega_{x}\right)}{\omega\left(\omega-\omega_{x}\right)}+\frac{\theta\left(\omega_{x}-\omega\right)}{\omega_{x}\left(\omega_{x}-\omega\right)}\right)\right]_{[\omega]+}+\frac{4 \theta\left(\omega_{x}-2 \omega\right)}{\left(\omega_{x}-\omega\right)^{2}}\right. \\
& \left.+4\left[\frac{\theta\left(2 \omega-\omega_{x}\right)}{\left(\omega_{x}-\omega\right)^{2}}\right]_{[\omega]++}-\frac{\delta\left(\omega_{x}-\omega\right)}{\omega}\left(\frac{1}{2} \ln ^{2} \frac{\mu_{H}^{2}}{\omega^{2}}-\ln \frac{\mu_{H}^{2}}{\omega^{2}}+\frac{3 \pi^{2}}{4}+2\right)\right\}-\left(3 \ln \frac{\mu_{H}}{m_{b}}+2\right) \delta\left(x-x_{\omega}\right),
\end{aligned}
$$

where for brevity we have introduced the shorthands

$$
x_{\omega} \equiv \frac{\omega}{m_{b}+\omega}, \quad \omega_{x} \equiv \frac{m_{b} x}{1-x},
$$

by noticing that the prefactor of the second term at the right-hand side of Eq. (14), we rewrite $m_{b} /(1-x)^{2}=d \omega_{x} / d x$. The " + " and " ++ " functions are understood in the distributive sense, whose exact definition can be found in [28]. The subscript $[x / \omega]$ enforces whether to convolute the plus function with a test function over $x$ or $\omega$. It is reassuring that $\mu_{Q}$ and $\mu_{H}$ dependence of $Z^{(1)}$ in Eq. (15) are explicitly compatible with the evolutions equations in Eqs. (2) and (5).

Our refactorization program has obvious strength to optimize the theoretical predictions for hard exclusive $B$ production processes. Plugging Eq. (9) into Eq. (1), one obtains

$$
\begin{aligned}
\mathcal{M}= & \int_{0}^{\infty} d \omega \mathcal{T}^{\operatorname{expd}}\left(\omega, \frac{Q}{m_{b}} ; \mu_{H}\right) \Phi_{+}^{\mathrm{HQET}}\left(\omega, \mu_{H}\right) \\
& +\mathcal{O}\left(\frac{m_{b}}{Q}, \frac{1}{m_{b}}\right)
\end{aligned}
$$

$$
\mathcal{T}^{\operatorname{expd}}\left(\omega, \frac{Q}{m_{b}} ; \mu_{H}\right)=\int_{0}^{1} d x T\left(x, \mu_{Q}\right) Z\left(x, \omega, m_{b} ; \mu_{Q}, \mu_{H}\right) .
$$

Equation (17) is the desired factorization formula that merges the virtues of both collinear and HQET factorization approaches, which is assumed to yield the most optimized prediction for hard exclusive $B$ production. As indicated in Eq. (17b), a more effective way of organizing calculation is to first utilize the existing knowledge on the hard-scattering kernel $T(x)$ (typically proportional to $1 / x$ ) in collinear factorization. Since the quark mass has been dropped, there is no difference for the hard-scattering kernel between $B$ and $\pi$ production. One then employs Eq. (17b) to obtain an effective hard-scattering kernel $\mathcal{T}^{\text {expd }}$. In accordance with the HQET factorization (17a), one can convolve this effective $\mathcal{T}^{\text {expd }}$ with $\Phi_{+}^{\mathrm{HQET}}$ to generate ultimate predictions. Notice that the $\mathcal{T}^{\text {expd }}$ is not identical with the $\mathcal{T}$ that arises from the literal fixedorder calculation in HQET factorization (4). Nevertheless, $\mathcal{T}^{\text {expd }}$ amounts to expanding $\mathcal{T}$ to lowest order in $m_{b} / Q$. 
Consequently, $\mathcal{T}$ expd can only depend on $Q / m_{b}$ logarithmically. We have explicitly verified that, by invoking Eq. (17), our $\mathcal{T}^{\text {expd }}$ indeed coincides with the expanded hard kernel $\mathcal{T}$ through order $\alpha_{s}$ for the $W \rightarrow B \gamma$ process [18].

Another remarkable merit of Eq. (17) is to expedite the resummation of the collinear logarithm $\alpha_{s} \ln \left(Q / m_{b}\right)$ to all orders. Since the $\mu_{Q}$ dependence of the $Z$ function is governed by the ERBL equation, we may follow the recipe outlined in [29] that also employs the ERBL equation to resum the leading collinear logarithm for exclusive quarkonium production, to recast Eq. (17b) into

$$
\begin{aligned}
& \mathcal{T}_{\mathrm{LL}}^{\text {expd }}\left(\omega, Q / m_{b} ; \mu_{H}\right)=\int_{0}^{1} d x T(x, Q) Z\left(x, \omega, m_{b} ; Q, \mu_{H}\right), \\
& =\int_{0}^{1} d x T^{(0)}(x) \exp \left[\kappa C_{F} V_{0} *\right] Z^{(0)}\left(x, \omega, m_{b}\right),
\end{aligned}
$$

where, in the second equation, we have substituted the schematic solution of the ERBL equation for $Z\left(x, \omega, m_{b} ; \mu_{Q}=Q\right)$, which is evolved from the initial IR scale at $\mu_{Q} \sim m_{b}$ to the UV scale $\mu_{Q} \sim Q$. The meaning of the "*" operation will become self-evident below. $\kappa$ is defined by

$$
\begin{aligned}
\kappa \equiv & \frac{2}{\beta_{0}} \ln \frac{\alpha_{s}\left(m_{b}^{2}\right)}{\alpha_{s}\left(Q^{2}\right)} \approx \frac{\alpha_{s}\left(Q^{2}\right)}{2 \pi} \ln \frac{Q^{2}}{m_{b}^{2}} \\
& +\beta_{0} \frac{\alpha_{s}^{2}\left(Q^{2}\right)}{(4 \pi)^{2}} \ln ^{2} \frac{Q^{2}}{m_{b}^{2}}+\cdots,
\end{aligned}
$$

where $\beta_{0}=\frac{11}{3} N_{c}-\frac{2}{3} n_{f}$ is the one-loop QCD $\beta$ function, and $n_{f}=5$ is the number of active quark flavors.

Equation (18) can be expanded iteratively [29],

$$
\begin{aligned}
\mathcal{T}_{\mathrm{LL}}^{\operatorname{expd}}\left(\omega, Q / m_{b} ; \mu_{H}\right)= & \int_{0}^{1} d x T^{(0)}(x) Z^{(0)}\left(x, \omega, m_{b}\right)+\kappa C_{F} \int_{0}^{1} d x \int_{0}^{1} d y T^{(0)}(x) V_{0}(x, y) Z^{(0)}\left(y, \omega, m_{b}\right) \\
& +\frac{\kappa^{2} C_{F}^{2}}{2} \int_{0}^{1} d x \int_{0}^{1} d y \int_{0}^{1} d z T^{(0)}(x) V_{0}(x, y) V_{0}(y, z) Z^{(0)}\left(z, \omega, m_{b}\right)+\cdots
\end{aligned}
$$

For a leading-twist (helicity-conserving) process, one typically bears $T^{(0)}(x) \propto 1 / x$. Substituting this together with Eq. (13) into Eq. (20), One can choose the order of multiple integration from the left to right, and leaves the integration over $Z^{(0)}$ in the last step. One then identifies the leading collinear logarithms at arbitrarily prescribed perturbative order. For example, at order $\alpha_{s}$, we obtain

$\mathcal{T}_{\mathrm{LL}}^{\operatorname{expd}}(\omega) \propto \frac{m_{b}}{\omega}\left\{1+\frac{\alpha_{s} C_{F}}{4 \pi} \ln \frac{Q^{2}}{m_{b}^{2}}\left(3+2 \ln \frac{\omega}{m_{b}}\right)+\left(\frac{\alpha_{s}}{4 \pi}\right)^{2} C_{F} \ln ^{2} \frac{Q^{2}}{m_{b}^{2}}\left[\frac{C_{F}}{2}\left(3+2 \ln \frac{\omega}{m_{b}}\right)^{2}+\frac{\beta_{0}}{2}\left(3+2 \ln \frac{\omega}{m_{b}}\right)\right]+\cdots\right\}$.

Reassuringly, the order- $\alpha_{s}$ collinear logarithm indeed coincides with the expanded NLO perturbative correction to the hard-scattering kernel in HQET factorization for the process $W \rightarrow B+\gamma$ [18], once $Q$ is identified with $m_{W}$. The coefficient of the order- $\alpha_{s}^{2}$ leading collinear logarithms awaits the confirmation by the future explicit two-loop calculation, which is certainly rather challenging. It is also possible, analogous to [29], to resum these leading collinear logarithms to all orders in $\alpha_{s}$ with the aid of some numerical recipes. We will explicitly illustrate the resummation for $W \rightarrow B\left(B^{*}\right)+\gamma$ in a long write-up.

Note that our refactorization framework is in a similar spirit to invoke the refactorization approach to recover the NRQCD short-distance coefficients associated with exclusive $B_{c}$ production through order $\alpha_{s}$, when expanded to the leading order in $m_{b} / Q$ [30,31].

In summary, in this Letter, we have established a novel factorization theorem (9) that connects two kinds of important LCDAs for heavy-flavor mesons through a perturbatively calculable coefficient function. This perturbative function has been determined through order $\alpha_{s}$. The physics underlying this factorization theorem looks quite lucid, just because HQET shares the identical IR behavior as QCD. It may look somewhat surprising why such a simple factorization formula has not been discovered until now. Based on this refactorization picture, we have devised a master formula, (17), tailored for tackling hard exclusive $B$ production processes. This factorization formula has inherited the virtues of both collinear and HQET factorization approaches, which is believed to generate the most optimized theoretical predictions. As a remarkable merit, this master formula also enables us to effectively resum large logarithms of type $\ln Q / m_{b}$ and $\ln m_{b} / \Lambda_{\mathrm{QCD}}$ in a controlled manner.

The work of S. I. and Y. J. is supported in part by the National Science Fund for Distinguished Young Scholars under Grant No. 11925506, by the National Natural 
Science Foundation of China under Grants No. 11875263 and No. 11621131001 (CRC110 by DFG and NSFC). S. I. also wishes to acknowledge the financial support from the CAS-TWAS President's Fellowship Program. The work of X.-N.X. is supported in part by the National Natural Science Foundation of China under Grant No. 11905296 and the Deutsche Forschungsgemeinschaft (Sino-German CRC 110). The work of D.-S. Y. is supported in part by the National Natural Science Foundation of China under Grants No. 11275263 and No. 11635009.

\section{APPENDIX: Subtlety in the moments of $B$ meson LCDAs}

The non-negative moments of the QCD LCDA are directly related to the matrix-elements of local QCD operators which are well-defined and finite. However, a famous symptom of the HQET LCDA is that its non-negative moments are UV divergent as indicated in a model-independent analysis given in [13]. For instance, its normalization is logarithmically divergent and can be regularized by imposing a UV cutoff $\Lambda$ on the integral,

$$
\begin{aligned}
\int_{0}^{\Lambda} d \omega \phi_{+}^{\mathrm{HQET}}\left(\omega, \mu_{H}\right)= & 1-\frac{\alpha_{s} C_{F}}{4 \pi}\left(\frac{1}{2} \ln ^{2} \frac{\mu_{H}^{2}}{\Lambda^{2}}+\ln \frac{\mu_{H}^{2}}{\Lambda^{2}}+\frac{\pi^{2}}{12}\right) \\
& +\mathcal{O}\left(\alpha_{s}^{2}\right)+\mathcal{O}\left(\frac{\Lambda_{\mathrm{QCD}}}{m_{b}}\right) . \quad(\mathrm{A} 1)
\end{aligned}
$$

Such defects of HQET LCDA may make one worry about the validity of the factorization formula that we propose in this work.

In this Appendix, we elaborate on how the LCDA moments defined in QCD and HQET are interrelated. We will show that the UV divergences in non-negative moments of HQET LCDA will not cause any trouble. We start with the first inverse moment of the QCD LCDA because it plays the most important role in phenomenological applications:

$$
\begin{aligned}
\left\langle\frac{1}{x}\right\rangle & \equiv \int_{0}^{1} \frac{d x}{x} \phi^{\mathrm{QCD}}\left(x, \mu_{Q}\right) \\
& =\int_{0}^{\infty} d \omega\left[\int_{0}^{1} \frac{d x}{x} Z\left(x, \omega ; \mu_{Q}, \mu_{H}\right) \phi_{+}^{\mathrm{HQET}}\left(\omega, \mu_{H}\right)\right] .
\end{aligned}
$$

Substituting the explicit expression of the $Z$ factor through $\mathcal{O}\left(\alpha_{s}\right)$ [Eqs. (13) and (15)] into Eq. (A2), and changing the variable $x \rightarrow \omega_{x} /\left(\omega_{x}+m_{b}\right)$, hence $d x\left[m_{b} /(1-x)^{2}\right] \rightarrow d \omega_{x}$, we then obtain

$$
\int_{0}^{1} \frac{d x}{x} Z^{(0)}\left(x, \omega, m_{b} ; \mu_{Q}, \mu_{H}\right)=\frac{1}{x_{\omega}}=\frac{m_{b}}{\omega}+1,
$$

$$
\begin{aligned}
\int_{0}^{1} \frac{d x}{x} Z^{(1)}\left(x, \omega, m_{b} ; \mu_{Q}, \mu_{H}\right)= & \frac{1}{2} \ln ^{2} \frac{\mu_{H}^{2}}{\Lambda^{2}}+\ln \frac{\mu_{H}^{2}}{\Lambda^{2}}+\frac{\pi^{2}}{12} \\
& +\frac{1}{x_{\omega}}\left[\left(3+2 \ln x_{\omega}\right) \ln \frac{\mu_{Q}^{2}}{\left(m_{b}+\omega\right)^{2}}-6\left(x_{\omega} \ln x_{\omega}+\bar{x}_{\omega} \ln \bar{x}_{\omega}\right)-2 \ln x_{\omega}+4 \operatorname{Li}_{2}\left(x_{\omega}\right)-2 \ln ^{2} x_{\omega}-\frac{2 \pi^{2}}{3}+6\right] \\
& +\frac{m_{b}}{\omega}\left(\frac{1}{2} \ln ^{2} \frac{\mu_{H}^{2}}{\omega^{2}}-\ln \frac{\mu_{H}^{2}}{\omega^{2}}+\frac{3 \pi^{2}}{4}-2\right)+\mathcal{O}\left(\frac{\omega}{\Lambda}\right)
\end{aligned}
$$

where $\Lambda$ represents the UV cutoff imposed on the upper limit of integration over $\omega_{x}$, just the same as the regulator for the normalization of the HQET LCDA in (A1).

Substituting (A3) into (A2) leads to

$$
\begin{aligned}
\left\langle\frac{1}{x}\right\rangle= & \int_{0}^{\infty} d \omega \frac{m_{b}}{\omega} \phi_{+}^{\mathrm{HQET}}\left(\omega, \mu_{H}\right) \\
& +\left[1+\frac{\alpha_{s} C_{F}}{4 \pi}\left(\frac{1}{2} \ln ^{2} \frac{\mu_{H}^{2}}{\Lambda^{2}}+\ln \frac{\mu_{H}^{2}}{\Lambda^{2}}+\frac{\pi^{2}}{12}\right)\right] \\
& \times \int_{0}^{\Lambda} d \omega \phi_{+}^{\mathrm{HQET}}\left(\omega, \mu_{H}\right)+\cdots \\
= & m_{b} \int_{0}^{\infty} \frac{d \omega}{\omega} \phi_{+}^{\mathrm{HQET}}\left(\omega, \mu_{H}\right)+1+\cdots
\end{aligned}
$$

The ellipses in Eq. (A4) denote the terms which may depend on $\Lambda$ but at a higher order in $\alpha_{s}$ and $\Lambda_{\mathrm{QCD}} / m_{b}$. One can see that the explicit $\Lambda$ dependence of the terms in the second line in Eq. (A4) gets canceled between the prefactor and normalization of HQET LCDA by implementing Eq. (A1). Therefore, we conclude that, despite the occurrence of the artificial cutoff $\Lambda$ in the intermediate step, the first inverse moment of the LCDA in QCD can be unambiguously related to its counterpart in HQET. In the practical applications, one usually neglects the unity in the line of Eq. (A4), since it is power suppressed with respect to the first inverse moment of the HQET LCDA. In this sense, we need only retain the leading term in $\omega / m_{b}$ expansion in Eq. (A3). It is worth remarking that we can obtain a relation between the first 
inverse moments of the QCD and HQET LCDAs similar to the one obtained in [20]. But we find that some additional logarithmic inverse moments are missed in [20].

For theoretical curiosity, one may also match the nonnegative moments of LCDA in QCD (albeit in absence of clear phenomenological interest) into the matrix elements of HQET operators. It turns out that the moments of QCD LCDA with non-negative powers can be related to the following convolution with HQET LCDA:

$$
\int_{0}^{\infty} d \omega\left(\frac{\omega}{\omega+m_{b}}\right)^{n} \phi_{+}^{\mathrm{HQET}}\left(\omega, \mu_{H}\right) .
$$

The above convolution possesses the identical degree of UV divergence as the normalization integral of $\phi_{+}^{\mathrm{HQET}}\left(\omega, \mu_{H}\right)$, because $\omega /\left(\omega+m_{b}\right) \sim 1$ as $\omega \gg m_{b}$. This UV divergence can be removed by following the same procedure that leads to Eq. (A4), and one can get finite non-negative moments for QCD LCDA from our factorization theorem. However, this is somewhat off the main topic of our Letter. We decide to leave a detailed analysis of this issue in the future work.

*saadiishaq@sns.nust.edu.pk

†jiay@ihep.ac.cn

*xnxiong@csu.edu.cn

§angds@ucas.ac.cn

[1] S. J. Brodsky and G. P. Lepage, Adv. Ser. Dir. High Energy Phys. 5, 93 (1989).

[2] G. P. Lepage and S. J. Brodsky, Phys. Rev. D 22, 2157 (1980).

[3] V. L. Chernyak and A. R. Zhitnitsky, Phys. Rep. 112, 173 (1984).

[4] G. P. Lepage and S. J. Brodsky, Phys. Lett. 87B, 359 (1979).

[5] A. V. Efremov and A. V. Radyushkin, Phys. Lett. 94B, 245 (1980).

[6] G. P. Korchemsky, D. Pirjol, and T. M. Yan, Phys. Rev. D 61, 114510 (2000).
[7] S. Descotes-Genon and C. T. Sachrajda, Nucl. Phys. B650, 356 (2003).

[8] E. Lunghi, D. Pirjol, and D. Wyler, Nucl. Phys. B649, 349 (2003); S. W. Bosch, R. J. Hill, B. O. Lange, and M. Neubert, Phys. Rev. D 67, 094014 (2003).

[9] M. Beneke, G. Buchalla, M. Neubert, and C. T. Sachrajda, Phys. Rev. Lett. 83, 1914 (1999).

[10] M. Beneke, G. Buchalla, M. Neubert, and C. T. Sachrajda, Nucl. Phys. B591, 313 (2000).

[11] A. G. Grozin and M. Neubert, Phys. Rev. D 55, 272 (1997).

[12] M. Beneke and T. Feldmann, Nucl. Phys. B592, 3 (2001).

[13] S. J. Lee and M. Neubert, Phys. Rev. D 72, 094028 (2005).

[14] B. O. Lange and M. Neubert, Phys. Rev. Lett. 91, 102001 (2003).

[15] E. Braaten, Y. Jia, and T. Mehen, Phys. Rev. D 66, 034003 (2002).

[16] E. Braaten, Y. Jia, and T. Mehen, Phys. Rev. D 66, 014003 (2002).

[17] E. Braaten, Y. Jia, and T. Mehen, Phys. Rev. Lett. 89, 122002 (2002).

[18] S. Ishaq, Y. Jia, X. Xiong, and D. S. Yang, Phys. Rev. D 100, 054027 (2019).

[19] Y. Grossman, M. König, and M. Neubert, J. High Energy Phys. 04 (2015) 101.

[20] V. Pilipp, arXiv:hep-ph/0703180.

[21] E. Eichten and B. R. Hill, Phys. Lett. B 234, 511 (1990).

[22] M. Neubert, Phys. Rep. 245, 259 (1994).

[23] M. Beneke and V. A. Smirnov, Nucl. Phys. B522, 321 (1998).

[24] J. P. Ma and Z. G. Si, Phys. Lett. B 647, 419 (2007).

[25] J. Xu and D. Yang, J. High Energy Phys. 07 (2016) 098.

[26] X. Ji, Phys. Rev. Lett. 110, 262002 (2013).

[27] X. Xiong, X. Ji, J. H. Zhang, and Y. Zhao, Phys. Rev. D 90, 014051 (2014).

[28] G. Bell and T. Feldmann, J. High Energy Phys. 04 (2008) 061.

[29] Y. Jia and D. Yang, Nucl. Phys. B814, 217 (2009).

[30] Y. Jia, J. X. Wang, and D. Yang, J. High Energy Phys. 10 (2011) 105.

[31] F. Feng, Y. Jia, and W. L. Sang, arXiv:1902.11288. 